\title{
A Sickle Cell Disease Patient with Severe Tricuspid Regurgitation and Early Developed Pulmonary Hypertension
}

\author{
Gaurang Vaidya ${ }^{1}$, Muhammad Sarwar ${ }^{2}$, Zongxia Sun $^{3}$, Tiemin $\mathrm{Wei}^{3}$ and Kan Liu ${ }^{1}$
}

\begin{abstract}
Pulmonary hypertension (PH) worsens the mortality of the patients with sickle cell disease (SCD). The exact mechanism of $\mathrm{PH}$ development/progression in SCD, including the role of tricuspid regurgitation (TR), remains unclear. We herein report an unusual SCD case, complicated by chronic thromboembolic disorder, who developed severe TR and an accelerated progression of PH. Tricuspid valve surgery significantly ameliorated the patient's symptoms and reduced hospital readmission. The early detection and management of the reversible disorder accelerating the PH development in SCD patients may alter the clinical course, improve the quality of life, and potentially affect the long-term outcome.
\end{abstract}

Key words: pulmonary hypertension, tricuspid regurgitation, sickle cell disease, thromboembolic disorder

(Intern Med 54: 2459-2462, 2015)

(DOI: 10.2169/internalmedicine.54.4463)

\section{Case Report}

A 35-year-old African American woman with a history of sickle cell disease (SCD), protein C deficiency and pulmonary embolism (PE) presented with worsening dyspnea on exertion (DOE) and lower extremity edema. She had been frequently admitted for DOE/chest pain, with a clinical diagnosis of "sickle cell crises (SCC)". On this physical examination, her blood pressure was $139 / 71 \mathrm{mmHg}$, pulse 82 bpm, $\mathrm{O}_{2}$ Sat $91 \%$ at room air, and body weight $191 \mathrm{lbs}$. She had jugular venous dilation, lower extremity edema, and a pan-systolic murmur (heard in the lower left sternal border). Abnormal laboratory findings included a low plasma hemoglobin level $(8.6 \mathrm{~g} / \mathrm{dL})$ and elevated pro-brain natriuretic peptide level $(7,500 \mathrm{pg} / \mathrm{mL})$. A chest X-ray showed clear lungs and an enlarged right atrium. An electrocardiogram revealed significant right axis deviation. Both the ventilation/ perfusion scan and chest computed tomographic angiography ruled out new PE. A transthoracic echocardiogram (TTE) revealed severe tricuspid regurgitation (TR) with right atrial dilatation (Fig. 1A, B), which raised a clinical suspi- cion of Ebstein's anomaly. Repeated TTE 6 month later suggested an increased tricuspid regurgitant jet velocity (TRV) and pulmonary hypertension (PH). During the transesophageal echocardiography study (Fig. 1C, D), although a patent foramen ovale (PFO) was observed (Fig. 1F), Ebstein's anomaly was excluded according to the morphology of the anterior leaflet of tricuspid valve (TV) and the insertion site of the septal leaflet of TV. The TV was severely calcified with a damaged (bifurcated) anterior cuspid (Fig. 1E). According to the patient's clinical condition and repeated echocardiographic examination, the structurally abnormal TV with severe TR was most likely the cause of the significantly worsened $\mathrm{PH}$, which was unlikely to be reversed by PH medical treatment alone. Furthermore, the existence of an atrial shunt raised the concern of paradoxical embolism. Therefore, tricuspid valve surgery was performed. An original attempt to repair the valve failed to significantly reduce the severity of TR, which necessitated a valve replacement. In addition, PFO closure was performed (Fig. 2A-D). The pathological examination revealed that both the valve and subvalvular apparatus were extremely calcified and foreshortened. Additionally, the anterior leaflet

${ }^{1}$ Department of Medicine, SUNY Upstate Medical University, USA, ${ }^{2}$ Department of Anesthesia, SUNY Upstate Medical University, USA and ${ }^{3}$ Department of Cardiology, Lishui Central Hospital, Zhejiang University, China

Received for publication November 10, 2014; Accepted for publication February 15, 2015

Correspondence to Dr. Kan Liu, liuk@upstate.edu 

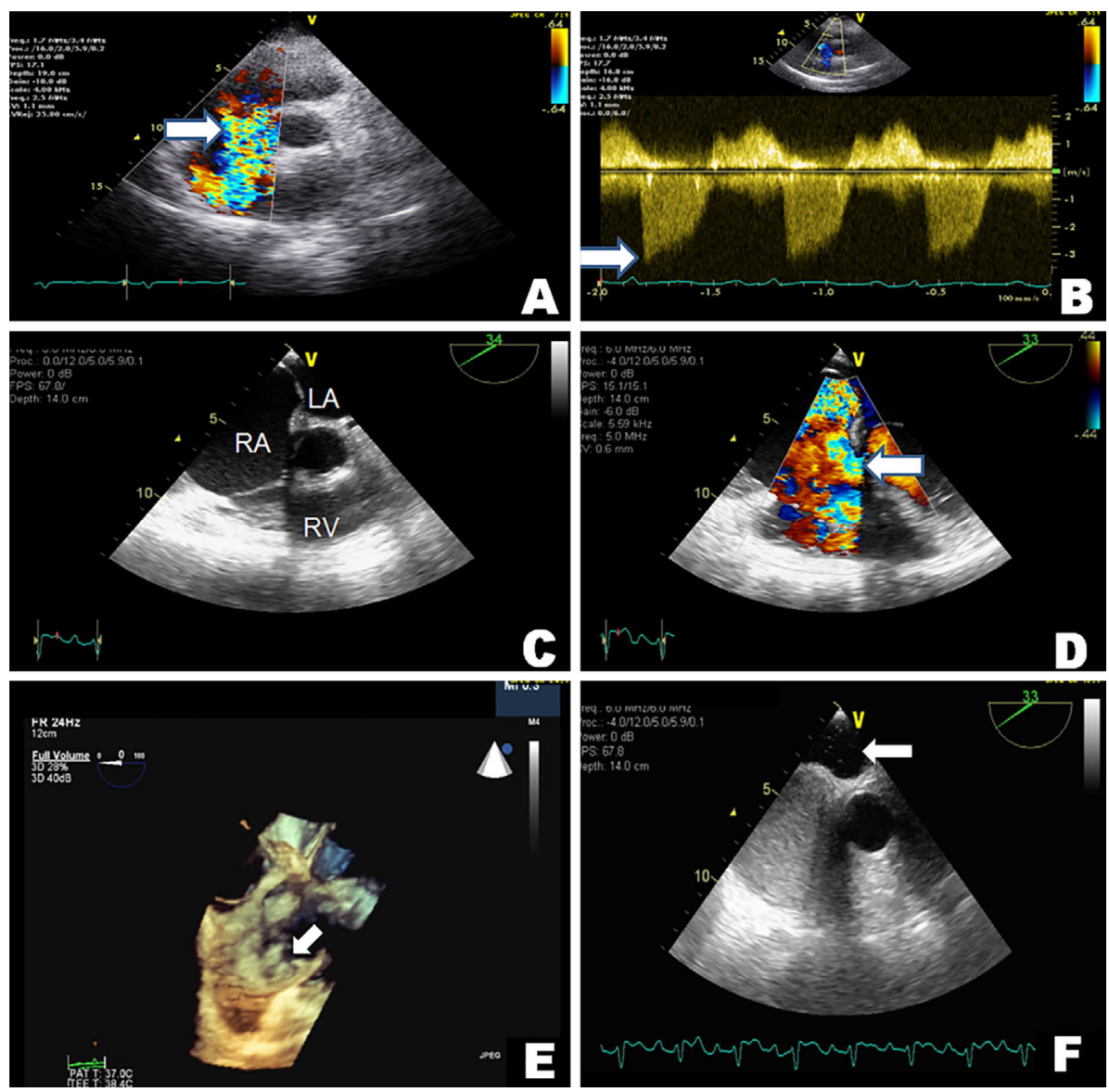

Figure 1. Tricuspid regurgitation (TR) in a patient with sickle cell disease (SCD) and chronic thromboembolic disorder. A: Color Doppler studies show severe TR (arrow) on the transthoracic echocardiogram (TTE). B: Doppler studies show severe TR (early-peaking TR flow jet) and PH (increased tricuspid regurgitant jet velocity, arrow) on the TTE. C: Remarkable right atrial dilatation shown on the TEE. D: Color Doppler studies show severe TR (arrow) on the TEE. E: Threedimensional reconstruction of the tricuspid valve (TV) on the TEE shows thickened leaflets and a damaged (bifurcated) anterior cuspid. F: A saline bubble study suggests a patent foramen ovale.

was severely damaged. After TVR, the patient's PH improved, and the sizes of the RA/RV were reduced (Fig. 2E-H). During the two-year follow-up, this patient has had only one hospitalization for SCC. Her physical exercise tolerance additionally improved. The repeated echocardiograms have consistently shown the amelioration of $\mathrm{PH}$.

\section{Discussion}

The TV damage with severe TR in this young SCD patient was not caused by Ebstein's anomaly. The postulated etiology was either previously unrecognized infective endocarditis from the recurrent blood transfusions for her SCD anemia or non-bacterial endocarditis/embolism from chronic thromboembolic events secondary to the protein $\mathrm{C}$ deficiency (1). According to her clinical and imaging data, the severe TR had likely become the most important contributor to the accelerated $\mathrm{PH}$ progression, clinical deterioration, and frequent hospital readmissions. Furthermore, the increased right ventricular pressure and transient right to left shunting through the PFO could have enhanced the respiratory diffi- culties and hypoxia $(2,3)$. Therefore, the timely management of TR became crucial.

$\mathrm{PH}$ develops in up to $30 \%$ of SCD patients and is considered to be a marker of early mortality $(4,5)$. The mechanisms underlying the PH development in SCD include pulmonary vasoconstriction secondary to reduced nitric oxide availability (scavenged by free hemoglobin), the release of arginase by hemolytic red blood cells, chronic hypoxia, iron overload, high cardiac output and asplenism $(6,7)$. Although increased echocardiography-derived TRV has long been considered to be an important marker for the PH development and increased mortality in SCD (8), the specific role of TR itself in the development and progression of $\mathrm{PH}$ in the patients with SCD has not yet been defined. Due to the increased pulmonary resistance and afterload in the SCD patients, the regurgitant volume of TR may increase disproportionately to the actual effective regurgitant orifice area of TR (9). This "functional" TR can increase the RV volume overload and structural remodeling, further worsening the structural abnormality of the TV. This "the worse, the worse" condition would certainly accelerate the PH develop- 


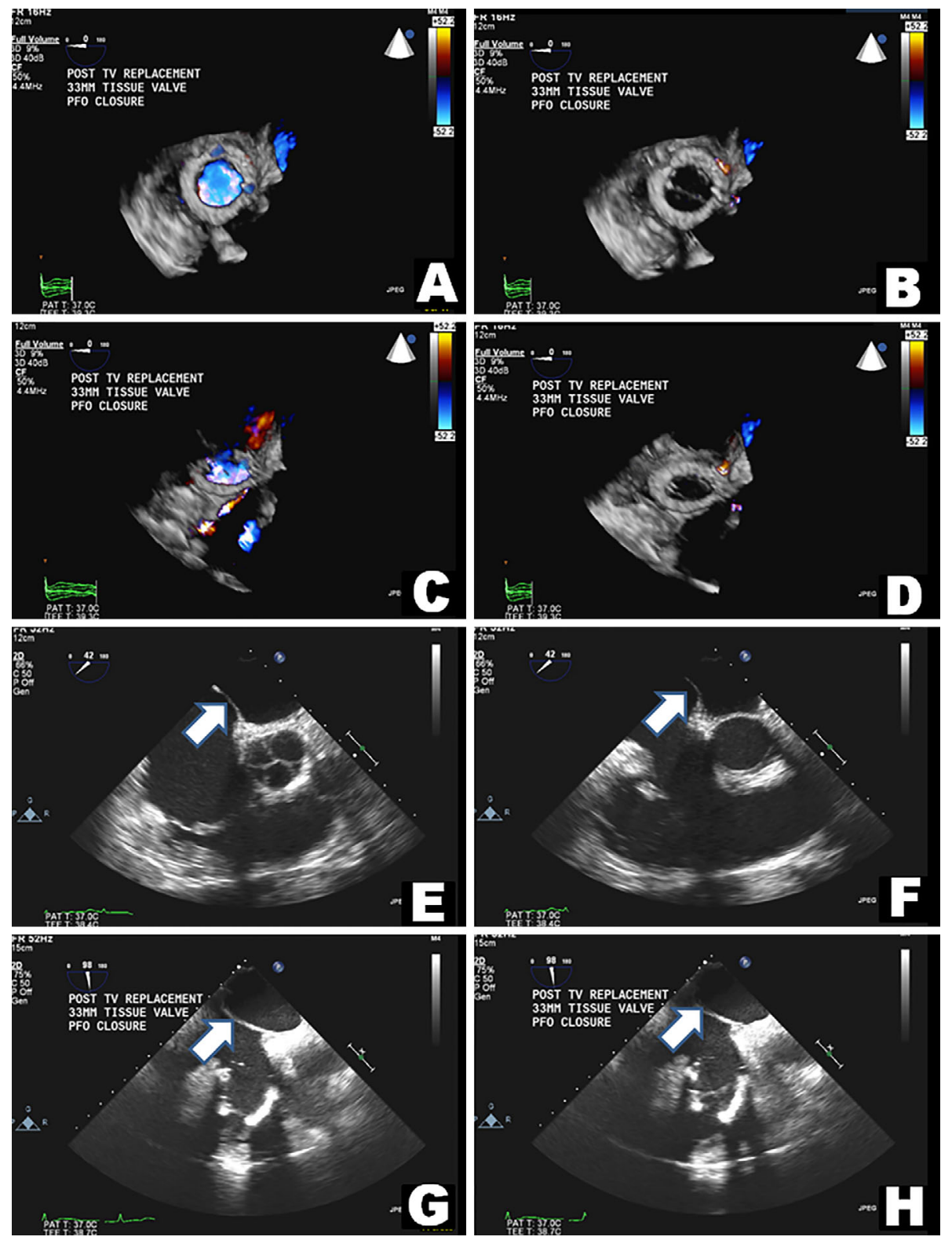

Figure 2. Surgery management of TR in a patient with SCD and chronic thromboembolic disorder. $A$ and B: Color Doppler studies on the TEE show a normal forward flow through the prosthetic valve post TV replacement (TVR) during diastole. C and D: Color Doppler in TEE studies demonstrate no residual TR through the prosthetic valve post TVR during systole. E-H: TEE studies show reduced right atrial/ventricular sizes after TVR. E and F: before TVR; G and H: after TVR. E and G: diastole; $\mathrm{F}$ and $\mathrm{H}$ : systole. The arrows indicate an improvement in the left atrial septum deviation after TVR.

ment in SCD. Although no consensus has currently been reached on the indications and timing of surgery for TR in SCD patients, the surgical correction of TR should be promptly advocated to break this vicious cycle in these "high-risk" patients, to decelerate the progression of $\mathrm{PH}$, and potentially to improve the long-term outcome.

The authors state that they have no Conflict of Interest (COI).

\section{References}

1. Ozkutlu S, Saraclar M, Atalay S, Demircin M, Ruacan S. Two- dimensional echocardiographic diagnosis of tricuspid valve noninfective endocarditis due to protein $\mathrm{C}$ deficiency (lesion mimicking tricuspid valve myxoma). Jpn Heart J 32: 139-145, 1991.

2. Auger WR, Kerr KM, Kim NH, Fedullo PF. Evaluation of patients with chronic thromboembolic pulmonary hypertension for pulmonary endarterectomy. Pulm Circ 2: 155-162, 2012.

3. Machado RF, Mack AK, Martyr S, et al. Severity of pulmonary hypertension during vaso-occlusive pain crisis and exercise in patients with sickle cell disease. Br J Haematol 136: 319-325, 2007.

4. Furlow B. Pulmonary hypertension of sickle cell disease: new guidelines. Lancet Respir Med 2: 263, 2014.

5. Klings ES, Machado RF, Barst RJ, et al. An official american thoracic society clinical practice guideline: Diagnosis, risk stratification, and management of pulmonary hypertension of sickle cell 
disease. Am J Respir Crit Care Med 189: 727-740, 2014.

6. Gladwin MT, Sachdev V, Jison ML, et al. Pulmonary hypertension as a risk factor for death in patients with sickle cell disease. $\mathrm{N}$ Engl J Med 350: 886-895, 2004.

7. Vichinsky EP. Pulmonary hypertension in sickle cell disease. N Engl J Med 350: 857-859, 2004.

8. Sachdev V, Kato GJ, Gibbs JS, et al. Echocardiographic markers of elevated pulmonary pressure and left ventricular diastolic dysfunction are associated with exercise intolerance in adults and adolescents with homozygous sickle cell anemia in the united states and united kingdom. Circulation 124: 1452-1460, 2011.

9. Boppana VS, Bhatta L, Liu K. Reversible TR in acute and chronic cardiomyopathy: medical versus surgical management? JACC Cardiovasc Imaging 6: 920-921, 2013.

(C) 2015 The Japanese Society of Internal Medicine http://www.naika.or.jp/imonline/index.html 